\title{
Clinical and laboratory characteristics of patients with thyroid diseases with and without alanine aminotransferase levels above the upper tertile - Cross-sectional analytical study
}

Nathanael de Oliveira e Silva', Marcelo Fernando Ronsoni², Bruno da Silveira Colombo ${ }^{3}$, Carina Gabriela Corrêa ${ }^{3}$, Simone Aiko Hatanaka', Maria Heloisa Büsi da Silva Canalli3 Leonardo de Lucca Schiavon', Janaína Luz Narciso-Schiavon'

\begin{abstract}
Objective: Thyroid disease affects $6.6 \%$ of the general population. The liver is fundamental in metabolizing thyroid hormones, and hepatocytes are often affected in thyroid disease. We aimed to compare clinical and laboratory parameters among thyroid disease patients with alanine aminotransferase (ALT) levels above vs. below the upper tertile. Subjects and methods: A retrospective cross-sectional analytical study was conducted in the endocrinology clinic at Polydoro Ernani de São Thiago University Hospital. Patients with thyroid disease between August 2012 and January 2014 were included in the study. Clinical and laboratory parameters were collected from medical records. Results: One hundred patients were included, of which $14.0 \%$ were male, with a mean age of 49.1 \pm 14.4 years. ALT levels ranged from 9 to $90 \mathrm{U} / \mathrm{L}$, and the ALT upper tertile was defined as 0,64 times the upper normal limit (xUNL). Patients with ALT levels above the upper tertile exhibited a higher proportion of systemic arterial hypertension (SAH), a higher mean abdominal circumference and a higher frequency of elevated TSH levels than did patients with ALT levels below the upper tertile. In multivariate analysis, ALT $\geq 0.64$ ( $x U N L$ ) was independently associated with abdominal circumference (odds ratio $[\mathrm{OR}]=0.087,95 \%$ confidence interval $[\mathrm{Cl}]$ 0012-0167, $\mathrm{P}=0.022$ ). $A L T(x U N L)$ correlated positively with total cholesterol $(r=0.213, P=0.042)$. Conclusions: In patients with thyroid diseases, it was observed that those with ALT above the upper tertile are associated with abdominal circumference and ALT levels correlate with total cholesterol. Arch Endocrinol Metab. 2016;60(2):101-7
\end{abstract}

Keywords

Alanine transaminase; thyroid diseases; thyrotropin; cholesterol; waist circumference
1 Divisão de Gastroenterologia Departamento de Medicina Interna, Universidade Federal de Santa Catarina (UFSC), Florianópolis, SC, Brazil ${ }^{2}$ Programa de Doutorado em Ciências Médicas, UFSC, Florianópolis, SC, Brazil ${ }^{3}$ Serviço de Endocrinologia, UFSC, Florianópolis, SC, Brazil

Correspondence to: Janaína Luz Narciso-Schiavon Departamento de Clínica Médica, HU Polydoro Ernani de São Thiago, Universidade Federal de Santa Catarina

Rua Professora Maria Flora Pausewang, $\mathrm{s} / \mathrm{n}^{\circ}, 3^{\circ}$ andar 88040-900 - Florianópolis, SC, Brazil janaina.narciso@uol.com.br

Received on Mar/31/2015 Accepted on May/6/2015

DOI: $10.1590 / 2359-3997000000066$

\section{INTRODUCTION}

$\mathrm{T}$ hyroid disease refers to a class of common disorders that are among the most prevalent medical afflictions. These disorders affect approximately 6.6\% of the world population, with females being 5-10 times more likely than males to develop some kind of abnormality in the thyroid gland $(1,2)$. The epidemiology of thyroid disease varies considerably between different subpopulations; living in iodine-deficient regions, which includes a third of the world population, is an important risk factor for the onset of goiter and hypothyroidism. The prevalence of hyperthyroidism is 0.5 $2 \%$ in women and $0.1-0.2 \%$ in men, whereas hypothyroidism occurs in $0.06-1.2 \%$ in women and $0.1-0.4 \%$ in men (2).
The thyroid gland synthesizes hormones that affect the functioning of all organs, tissues and cells of the body. Thyroid hormones modulate oxygen consumption rates, thermogenesis, the expression of the low-density lipoprotein (LDL) receptor, the strength and frequency of myocardial contraction ( 3 ) and bone turnover (4). In addition, thyroid hormones have great importance in the physiology of the gastrointestinal tract: they are necessary for the maturation of its mucous membranes and influence gastrointestinal motility, glucose and fat uptake, and the composition of bile salts (5).

Besides being vulnerable to thyroid afflictions, the liver is fundamental in metabolizing thyroid hormones and regulates their systemic effects $(6,7)$. The de-iodin- 
ation and conversion of thyroxin (T4) to triiodothyronin (T3) also occurs in the liver $(8,9)$. The liver synthesizes several plasma proteins that bind to lipophilic thyroid hormones, keeping the great majority inactive at any given time (10). Therefore, while the thyroid is important for maintaining the integrity of liver cells, the integrity of hepatocytes is essential for maintaining the homeostasis of thyroid hormones' functions.

Hypothyroidism directly affects the structure and function of hepatocytes, and is associated with cholestatic jaundice, which is attributed to reduced excretion of bilirubin and bile and reduced flow of bile (10). In addition, hypothyroidism is associated with obesity and dyslipidemia (11), which can induce steatogenesis and lead to nonalcoholic steatohepatitis. This association occurs because thyroid hormones increase the expression of LDL receptors in hepatocytes (12) and increase the activity of fat-reducing liver enzymes, leading to decreased levels of circulating LDL (13). Hypothyroidism also leads to decreased intestinal motility that promotes increased intestinal absorption of enteric cholesterol (14). Indeed, increased serum levels of gamma-glutamyl transferase (GGT) and alanine aminotransferase (ALT) have been detected even in cases of minimal hypothyroidism. Moreover, liver damage can be detected in subclinical thyroid disease cases, with evidence of metabolic changes in liver-associated laboratory parameters (15).

An excess of thyroid hormone is also associated with liver injury. In thyrotoxicosis, hepatic afflictions are common and include hepatocellular injury, elevated liver enzymes (aspartate aminotransferase [AST] and ALT), cholestasis, and increased levels of alkaline phosphatase (ALP), GGT and bilirubin. It is believed that hepatitis caused by thyrotoxicosis is due to hypoxia in perivenular regions, reflecting an increased oxygen uptake by hepatocytes without a corresponding increase in blood flow (16).

Since serum ALT concentration is the most commonly used variable for assessment of liver disease (15) and is a is a suitable test to identify subjects with chronic liver disease even in an asymptomatic phase (16), we aim to compare clinical and laboratory characteristics among individuals with thyroid disease with and without ALT above the upper tertile.

\section{SUBJECTS AND METHODS}

This cross-sectional analytical study was comprised of adults with thyroid diseases treated at outpatient clinics of the endocrinology unit of the University Hospital of Federal University of Santa Catarina. On routine visits, patients were invited to take part in the study and sign the informed consent form in duplicate. Clinical and laboratory data were collected from the patients' medical records. Individuals with thyroid disease treated between August 2012 and January 2014 were eligible for inclusion in the study. Patients who didn't take tests to assess liver (ALT and AST) or thyroid (thyroid-stimulating hormone $[\mathrm{TSH}]$ and T4 free) function or who had insufficient information in their medical record were excluded from the study.

Patients were analyzed for the following clinical and epidemiological parameters: age, gender, co-morbidities (diabetes mellitus, systemic arterial hypertension, hypercholesterolemia hypertriglyceridemia), ethnicity, weight, body mass index, waist circumference, thyroid disease type, thyroid hormone metabolism-related medication use, prior ablation of the thyroid with radioactive iodine $\left(\mathrm{I}^{131}\right)$ and prior surgical thyroidectomy. Patients were considered to have diabetes mellitus if they displayed symptoms of polyuria, polydipsia and unexplained weight loss plus fasting glucose $>126 \mathrm{mg} / \mathrm{dL}$ and/or plasma glucose $>200 \mathrm{mg} / \mathrm{dL}$ after 2 hours of $75 \mathrm{mg}$ of glucose administration (17). Patients were considered hypertensive if their blood pressure was $\geq$ $140 / 90 \mathrm{mmHg}$ (18). Patients were considered to have dyslipidemia if they displayed isolated hypercholesterolemia (defined as total serum cholesterol $\geq 200 \mathrm{mg}$ / $\mathrm{dL}$, LDL cholesterol $\geq 160 \mathrm{mg} / \mathrm{dL}$ ) and/or low levels of high density lipoprotein (HDL) $(<50 \mathrm{mg} / \mathrm{dL}$ for women and $<40 \mathrm{mg} / \mathrm{dL}$ for men) and/or isolated hypertriglyceridemia (defined as serum triglycerides $\geq$ $150 \mathrm{mg} / \mathrm{dL}$ ) (19). Laboratory parameters analyzed were: fasting glucose, fasting insulin, total cholesterol, HDL, LDL, triglycerides, AST, ALT, free T4, TSH, surface antigen of the hepatitis B virus and antibodies to hepatitis $\mathrm{C}$ virus. Laboratory tests were expressed in absolute values.

Patients were evaluated in relation to ALT values. Bivariate analyses were performed to identify parameters associated with ALT values above the upper tertile. Numerical parameters were expressed as mean \pm standard deviation and compared using Student's t test for samples with normal distribution or Mann-Whitney test for non-normally distributed samples. Qualitative parameters were represented by relative frequency (\%) and analyses performed using chi-squared (q) test or, when necessary, Fisher exact test (f). Bivariate and mul- 
tivariate analyses were performed to identify parameters independently associated with ALT above the upper tertile. The correlation between continuous parameters and ALT was assessed using Spearman's correlation coefficient. P-values less than 0.05 were considered statistically significant. All tests were two-tailed and performed by the Statistical Package for Social Sciences (SPSS) version 17.0 (SPSS, Chicago, Illinois, USA).

This study protocol conforms to the ethics recommendations of the Helsinki Declaration of 1975 and was approved by the Ethics and Human Research Committee of the university, number 318.763 .

\section{RESULTS}

\section{Characteristics of the patient population}

One hundred and thirteen patients with thyroid disease treated at this institution were evaluated for inclusion in the study. Thirteen patients were excluded from the study for not having conducted tests of ALT, AST, free T4 and/or TSH.

The mean age of the 100 patients included in the study was $49.1 \pm 14.4$ years. Fourteen percent of the patients were men. The majority of patients $(71 \%)$ had been diagnosed with Hashimoto's thyroiditis, $11.8 \%$ with unspecified hypothyroidism, $2.1 \%$ with unspecified hyperthyroidism, $6.5 \%$ after ablation with radioactive iodine $\left(\mathrm{I}^{131}\right), 4.3 \%$ after surgical thyroidectomy and $4.3 \%$ with Graves' disease. On average, patients had $1.2 \pm 0.3 \mathrm{ng} / \mathrm{dL}$ of free T4 and $26.1 \pm 8.2 \mathrm{mIU} / \mathrm{mL}$ of TSH. When taken, the mean dose of levothyroxine was $96.8 \pm 42.0 \mu \mathrm{g}$.

The mean ALT level was $36.3 \pm 11.8 \mathrm{U} / 1$. The upper tertile of ALT was $0.64 \mathrm{xUNL} ; 35$ of the $100 \mathrm{pa}$ tients had an ALT level greater than this upper tertile. On average, patients had ALT values of $0.6 \pm 0.2(0.6)$ $\mathrm{xUNL}$, a value below the upper tertile of serum concentrations of the enzyme. Two patients had hepatitis $\mathrm{C}$ and none were positive for hepatitis $\mathrm{B}$ virus surface antigen. Regarding liver biochemistry, individuals studied had a mean ALT of $0.6 \pm 0.2(0.6) \times \mathrm{UNL}$; AST of $0.6 \pm 0.2(0.6) \times U N L ; F A$ of $0.7 \pm 0.2(0.6) \times U N L$; GGT $0.7 \pm 0.6(0.5)$ xUNL. Of the studied individuals $35(35 \%)$ had ALT equal to or above the upper tertile (0.64 xUNL).

\section{Comparative analysis of the patients above and below the ALT upper tertile}

When the 35 patients with ALT levels above the upper tertile were compared to the 65 patients with ALT levels below the upper tertile (Tables 1 and 2), we observed a higher proportion of individuals with systemic arte-

Table 1. Clinical parameters of 100 patients with thyroid disease according to ALT values above the upper tertile*

\begin{tabular}{|c|c|c|c|c|}
\hline Characteristic & All $(n=100)$ & ALT $\geq 0.64(n=35)$ & ALT $<0.64(n=65)$ & $p$ \\
\hline Age (years) $^{\dagger}$ & $49.1 \pm 14.4(50.0)$ & $50.5 \pm 11.4(52.0)$ & $48.3 \pm 15.8(49.0)$ & $0.411^{t}$ \\
\hline Male (\%) & 14.0 & 5.7 & 18.5 & $0.129^{f}$ \\
\hline Arterial hypertension (\%) & 38.4 & 52.9 & 30.8 & $0.031^{q}$ \\
\hline Diabetes mellitus (\%) & 14.0 & 17.1 & 12.3 & $0.553^{f}$ \\
\hline Hypercholesterolemia (\%) & 36.0 & 40.0 & 33.8 & $0.541^{q}$ \\
\hline Hypertriglyceridemia (\%) & 16.0 & 25.7 & 10.8 & $0.052^{\mathrm{q}}$ \\
\hline Caucasian ethnicity (\%) & 92.7 & 93.8 & 92.2 & $1.000^{f}$ \\
\hline Weight $(\mathrm{kg})^{\dagger}$ & $70.5 \pm 14.2(68.9)$ & $71.3 \pm 13.3(70.5)$ & $70.0 \pm 14.7(68.0)$ & $0.661^{t}$ \\
\hline $\mathrm{BMI}\left(\mathrm{kg} / \mathrm{m}^{2}\right)^{\dagger}$ & $27.8 \pm 5.2(27.1)$ & $28.4 \pm 5.3(27.2)$ & $27.5 \pm 7.2(27.1)$ & $0.391^{\mathrm{m}}$ \\
\hline Waist circumference $(\mathrm{cm})^{\dagger}$ & $92.9 \pm 12.0(92.0)$ & $101.6 \pm 13.8(104.0)$ & $90.7 \pm 10.7(91.0)$ & $0.013^{t}$ \\
\hline Hyperthyroidism (\%) & 5.0 & 5.7 & 4.6 & $1,000^{\dagger}$ \\
\hline Hypothyroidism (\%) & 93.0 & 91.4 & 93.8 & $0.693^{\dagger}$ \\
\hline Thyroid medication (\%) & 93.9 & 91.2 & 95.3 & $0.415^{\dagger}$ \\
\hline Levothyroxine (\%) & 89.8 & 88.2 & 90.6 & $0.735^{\dagger}$ \\
\hline Levothyroxine dose $(\mathrm{mcg})^{\dagger}$ & $96.8 \pm 42.0(100.0)$ & $88.8 \pm 46.0(81.5)$ & $101.1 \pm 39.5(100.0)$ & $0.194^{t}$ \\
\hline Tiamazol (\%) & 4.3 & 3.0 & 4.9 & $1,000^{f}$ \\
\hline Previous ablation with I $\mathrm{I}^{131}$ & 8.0 & 2.9 & 10.8 & $0.255^{\dagger}$ \\
\hline Previous thyroidectomy (\%) & 4.0 & 0.0 & 6.2 & $0.295^{\dagger}$ \\
\hline
\end{tabular}


Table 2. Laboratory parameters of 100 patients with thyroid disease according to ALT values above the upper tertile*

\begin{tabular}{lcccc}
\hline Characteristic & $\begin{array}{c}\text { All } \\
(\mathbf{n = 1 0 0})\end{array}$ & $\begin{array}{c}\text { ALT } \geq \mathbf{0 . 6 4} \\
(\mathbf{n}=\mathbf{3 5})\end{array}$ & $\begin{array}{c}\text { ALT }<\mathbf{0 . 6 4} \\
(\mathbf{n}=\mathbf{6 5})\end{array}$ & $\boldsymbol{p}$ \\
\hline Free $\mathrm{T}_{4}(\mathrm{ng} / \mathrm{dL})$ & $1.2 \pm 0.3(1.2)$ & $1.2 \pm 0.3(1.2)$ & $1.2 \pm 0.3(1.2)$ & $0.768^{\mathrm{m}}$ \\
$\mathrm{TSH} \geq 3.0 \mu \mathrm{Ul} / \mathrm{mL}(\%)$ & 52.0 & 65.7 & 44.6 & $0.044^{\mathrm{q}}$ \\
Glucose $(\mathrm{mg} / \mathrm{dL})^{\dagger}$ & $103.5 \pm 37.6(93.0)$ & $106.8 \pm 33.0(95.0)$ & $101.9 \pm 39.9(92.0)$ & $0.316^{\mathrm{m}}$ \\
Fasting insulin $(\mu \mathrm{U} / \mathrm{mL}) \#^{\dagger}$ & $9.7 \pm 12.2(8.6)$ & $7.8 \pm 4.4(7.0)$ & $10.9 \pm 15.0(8.6)$ & $0.431^{\mathrm{m}}$ \\
Total colesterol $(\mathrm{mg} / \mathrm{dL})^{\dagger}$ & $202.5 \pm 48.3(195.0)$ & $213.6 \pm 48.7(203.0)$ & $197.0 \pm 47.5(190.5)$ & $0.070^{\mathrm{m}}$ \\
HDL $(\mathrm{mg} / \mathrm{dL})^{\dagger}$ & $53.3 \pm 13.8(50.5)$ & $54.4 \pm 14.4(53.0)$ & $52.7 \pm 13.6(50.0)$ & $0.418^{\mathrm{m}}$ \\
$\left.\mathrm{LDL}^{\dagger} \mathrm{mg} / \mathrm{dL}\right)^{\dagger}$ & $123.2 \pm 38.8(121.0)$ & $131.5 \pm 43.3(124.0)$ & $119.0 \pm 35.8(116.5)$ & $0.112^{\mathrm{m}}$ \\
Triglycerides $(\mathrm{mg} / \mathrm{dL})^{\dagger}$ & $112.2 \pm 66.0(93.0)$ & $130.7 \pm 80.3(114.0)$ & $103.1 \pm 53.6(88.0)$ & $0.114^{\mathrm{m}}$ \\
\hline
\end{tabular}

${ }^{*}$ ALT $\geq 0.64$ times the upper normal limit (XUNL); ${ }^{\dagger}$ mean \pm standard deviation (median); ${ }^{~}$ data available for 66 patients; Free T4: free thyroxine; TSH: thyroid-stimulating hormone; HDL: high-density lipoprotein; LDL: low-density lipoprotein; m: Mann Whitney; q: Chi-square test.

rial hypertension $(52.9 \%$ versus $30.8 \% ; \mathrm{P}=0.031)$ and with TSH levels $\geq 3.0 \mathrm{mIU} / \mathrm{ml}(65.7 \%$ versus $44.6 \%$; $\mathrm{P}=0.044)$. A higher mean waist circumference was also observed among the patients with ALT levels above the upper tertile $(101.6 \pm 13.8 \mathrm{~cm}$ versus $90.7 \pm 10.7 \mathrm{~cm}$; $\mathrm{P}=0.013)$. There were no significant differences between the two groups in age, weight, glucose, free T4, fasting insulin, HDL, LDL or triglycerides. There were also no differences in gender, ethnicity, history of diabetes, hypercholesterolemia, hyperthyroidism, hypothyroidism, use of thyroid medication, prior ablation with $\mathrm{I}^{131}$ or prior surgical thyroidectomy.

Parameters with $\mathrm{P}$ values $<0.050$ (waist circumference, systemic arterial hypertension frequency and elevated TSH frequency) were included in a multivariate analysis. Only waist circumference $(\mathrm{OR}=0.087$, 95\% CI0.012-0.167, $\mathrm{P}=0.022$ ) was associated independently with ALT levels.

There was also a positive correlation between ALT levels and total cholesterol levels across the entire study sample, independent of ALT level strata $(\mathrm{r}=0.213$, $\mathrm{P}=0.042$ ) (Figure 1). No such correlation between ALT levels and age, waist circumference, weight, body mass index, HDL, LDL, triglycerides, fasting insulin, fasting glucose, free T4 nor TSH was observed.

\section{DISCUSSION}

With respect to the parameters of age $(20,21)$ gender $(22)$, and etiology of thyroid disease $(23,24)$, the present study sample is consistent with previous studies of thyroid disease. The average age reported in individuals

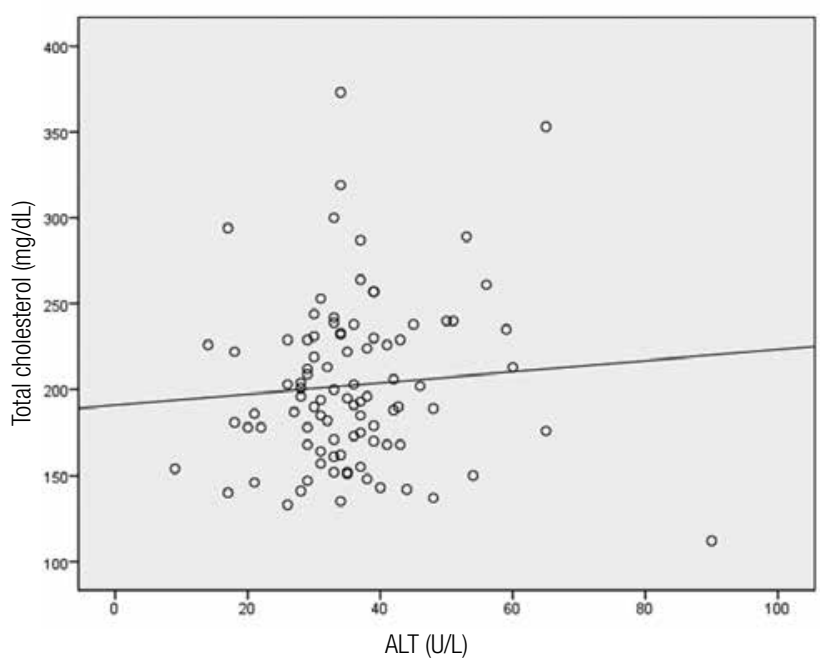

Figure 1. Study patients present a positive correlation between total cholesterol levels and ALT levels ( $r=0.213 ; p=0.042$ ).

with thyroid disease is $41-50$ years $(20,21)$, and the prevalence of hypothyroidism increases with increasing age of the population studied (25). Among our patients, 71\% had Hashimoto's thyroiditis as the etiology of thyroid disease, which corroborates findings in the literature that this thyroiditis ranks as the leading cause of hypothyroidism $(23,24)$.

Liver disease is the primary cause of increased ALT levels. Several factors other than liver disease must be considered when interpreting ALT levels, such as: $45 \%$ variation during the day; $10-30 \%$ variation from one day to the next; $15 \%$ elevation in African-American men; a direct relationship between weight and ALT; and a three-fold increase with strenuous exercise (26). Dif- 
ferential diagnosis on aminotransferases elevations may reflect liver diseases (alcoholic and nonalcoholic hepatic steatosis, liver injury induced by drugs, viral hepatitis, autoimmune hepatitis, hemochromatosis, etc.) or be caused by pathologies affecting organs other than the liver such as thyroid disease, celiac disease, hemolysis, muscle disorders, among others (27). Since blood was collected in the morning, before breakfast, fasting may have controlled some variables that would have influenced the ALT levels. However, as in other studies, it was impossible for us to control all of the variables. The majority $(93 \%)$ of the patients in our study were Caucasian, and co-morbidities did not statistically influence the ALT levels.

The vast majority of individuals analyzed in this study presented ALT levels within the normal range. A recent study of the general population demonstrated that patients with normal versus elevated ALT levels may have a similar severity of nonalcoholic steatohepatitis, suggesting that ALT levels may sometimes be a misleading parameter for guiding clinical management (28). ALT levels may also be normal in patients with liver disease, so a revision of the normal range for ALT levels may be advisable (29). It was observed in bivariate analysis that ALT levels above the upper tertile are associated with hypertension, TSH levels $\geq 3.0 \mathrm{mIU} / \mathrm{ml}$ and increased waist circumference. This last parameter was also associated with ALT levels above the upper tertile in multivariate analysis. Furthermore, ALT levels correlated with total cholesterol levels independently of ALT level strata.

A higher proportion of patients with arterial hypertension was observed among patients with ALT levels above the upper tertile. Hanley and cols. (30), studying 633 individuals without diabetes or metabolic syndrome, also observed an increase in ALT levels in persons with SAH compared to those with normal blood pressure. Similar data were presented by Hong and cols. (31) when analyzing 2,074 women: the incidence of arterial hypertension was $37.32 \%$ among women with ALT levels $<20 \mathrm{U} / 1$, but $57.33 \%$ among women with ALT levels > $50 \mathrm{U} / \mathrm{l}(\mathrm{P}<0.01)$. Like increased ALT levels, hypertension is associated with thyroid disorders - whether they cause hypothyroidism or hyperthyroidism. When comparing patients with normal thyroids against those with hypothyroidism, Saito and cols. (32) observed that $5.5 \%$ of euthyroid patients had hypertension, compared to $14.8 \%$ in the hypothyroidism group. A similar situation was found in patients with hyperthyroidism. However, an increase in systolic pressure as the sole treatment of hyperthyroidism is capable of lowering blood pressure levels (33).

These results are in line with the descriptions in the medical literature indicating that both hyperthyroidism and hypothyroidism are associated with hepatic afflictions. Kubota and cols. (34), when analyzing patients with untreated Graves' disease or untreated painful thyroiditis, observed increases in liver enzyme levels compared with the control group. Mean ALT levels were $30.8 \mathrm{U} / 1,47.2 \mathrm{U} / \mathrm{l}$ and $17.4 \mathrm{U} / \mathrm{l}$ for the Graves' disease, thyroiditis and control groups, respectively.

We observed a significantly greater mean waist circumference in the group with ALT levels above the upper tertile than in the group with lower ALT levels. Data in the medical literature show a similar association. Individuals with ALT levels $<20 \mathrm{U} / 1$ exhibited a $34.25 \%$ prevalence of central obesity (waist circumference $\geq 90 \mathrm{~cm}$ for men and $\geq 80 \mathrm{~cm}$ for women), whereas individuals with ALT levels $>50 \mathrm{U} / 1$ exhibited a $67.16 \%$ prevalence of central obesity (31). Another study showed that patients with central obesity had a mean ALT level of $20.51 \mathrm{U} / 1$, whereas individuals without an increased waist circumference had a mean ALT level of $15.39 \mathrm{U} / \mathrm{l}$ (30). Shantha and cols. (35), studying the association between metabolic syndrome and the prevalence of hypothyroidism, noted that among patients in the study group (mean waist circumference of $100.1 \pm 5.72 \mathrm{~cm}), 21.9 \%$ had subclinical hypothyroidism and $7.4 \%$ had overt hypothyroidism. In the control group (mean waist circumference 84.2 $\pm 6.91 \mathrm{~cm}), 6.6 \%$ had subclinical hypothyroidism and $2.2 \%$ had overt hypothyroidism.

In this study, when we analyzed the relationship between serum cholesterol and ALT levels, there was a continuous correlation between the two parameters. Zhang and cols. (36) studied 1,214 people in China and found that those with metabolic syndrome had a mean cholesterol level of $197.55 \mathrm{mg} / \mathrm{dL}$ and a mean ALT level of $37.9 \mathrm{U} / 1$. In contrast, individuals without metabolic syndrome had a mean cholesterol level of $173.19 \mathrm{mg} / \mathrm{dL}$ and a mean ALT level of $27.7 \mathrm{U} / \mathrm{l}$. In a separate study, Kaliaperumal and cols. (21) found that patients with untreated hypothyroidism had a mean cholesterol level $(232.12 \pm 5.12 \mathrm{mg} / \mathrm{dL})$ significantly higher than the control group $(169.65 \pm 2.59 \mathrm{mg} / \mathrm{dL})$. A third study showed that individuals with TSH levels < $0.1 \mathrm{mIU} / \mathrm{ml}$ had a mean cholesterol level of $181.70 \mathrm{mg} / \mathrm{dL}$, whereas individuals with TSH levels $>10 \mathrm{mIU} / \mathrm{ml}$ had a mean cholesterol level of $209.15 \mathrm{mg} / \mathrm{dL}$ (15). 
The present study has some limitations to be mentioned. First, the number of patients is not significant considering the high prevalence of thyroid disorders in the general population. However, the University Hospital is a referral center for thyroid disorders and serves individuals throughout the state of Santa Catarina, which is one of the smaller states in Brazil. Additionally, the study is similar in size to other studies of thyroid disease patients. This was not a prospective study involving patients in the first outpatient evaluation. Rather, this was a cross-sectional study that included data from patients already undergoing medical treatment or even surgery. Thus, this study reflects patients in the "real world", with the disease under control or being controlled. The fact that information on alcohol and drug use was not available for analysis represents another limitation, as alcohol and drug use are well known to affect liver function. Nonetheless, other possible causes of liver disease and co-morbidities were analyzed and showed no influence on ALT levels.

Patients with thyroid disease, when being treated by specialist clinicians, are mostly clinically compensated. In such individuals, ALT levels correlated with total cholesterol levels and abdominal circumference associated independently with ALT levels above the upper tertile. Elevations in ALT level, cholesterol level and waist circumference in patients with compensated thyroid disease possibly reflect what is observed in the general population: the presence of metabolic syndrome as a risk factor for nonalcoholic steatohepatitis. It is possible that the group of patients with ALT above the upper tertile may benefit from dietary guidelines and weight management, as well as tracking of hepatic steatosis by ultrasound. However, these activities must be defined in future studies.

Acknowledgments: paper presented as a requirement for obtaining the Medical Doctor (MD) degree from the Federal University of Santa Catarina (UFSC), and it was presented at the " $31^{\circ}$ Congresso Brasileiro de Endocrinologia e Metabologia (CBEM)" as a poster.

Financial support: no financial support.

Disclosure: no potential conflict of interest relevant to this article was reported.

\section{REFERENCES}

1. Wu P. Thyroid disease and diabetes. Clin Diabetes. 2000;18(1): 38-9.
2. Vanderpump MP. The epidemiology of thyroid disease. Br Med Bull. 2011;99:39-51.

3. Klein I, Ojamaa K.Thyrotoxicosis and the heart. Endocrinol Metab Clin North Am. 1998;27(1):51-62.

4. Mosekilde L, Eriksen EF, Charles P. Effects of thyroid hormones on bone and mineral metabolism. Endocrinol Metab Clin North Am. 1990;19(1):35-63.

5. MiddletonWR. Thyroid hormones and the gut. Gut. 1971;12(2):172-7.

6. Hassi J, Sikkilä K, Ruokonen A, Leppäluoto J.The pituitary-thyroid axis in healthy men living under subarctic climatological conditions. J Endocrinol. 2001;169(1):195-203.

7. Larsen PR.Thyroidal triiodothyronine and thyroxine in Graves' disease: correlation with presurgical treatment, thyroid status, and iodine content. J Clin Endocrinol Metab. 1975;41(06):1098-104.

8. Bianco AC, Salvatore D, Gereben B, Berry MJ, Larsen PR. Biochemistry, cellular and molecular biology, and physiological roles of the iodothyronine selenodeiodinases. Endocr Rev. 2002;23(1):38-89.

9. Mendel CM, Cavalieri RR, Weisiger RA. Uptake of thyroxine by the perfused rat liver: implications for the free hormone hypothesis. Am J Physiol. 1988;255(2 Pt 1):E110-9.

10. Van Steenbergen W, Fevery J, De Vos R, Leyten R, Heirwegh KP, De Groote J. Thyroid hormones and the hepatic handling of bilirubin. I. Effects of hypothyroidism and hyperthyroidism on the hepatic transport of bilirubin mono- and diconjugates in the Wistar rat. Hepatology. 1989;9(2):314-21.

11. Zhang J, Jiang R, Li L, Li P, Li X, Wang Z, et al. Serum thyrotropin is positively correlated with the metabolic syndrome components of obesity and dyslipidemia in chinese adolescents. Int $J$ Endocrinol. 2014;2014:289503.

12. Ness GC, Lopez D, Chambers CM, Newsome WP, Cornelius P, Long $\mathrm{CA}$, et al. Effects of L-triiodothyronine and the thyromimetic L-94901 on serum lipoprotein levels and hepatic low-density lipoprotein receptor, 3-hydroxy-3-methylglutaryl coenzyme A reductase, and apo A-I gene expression. Biochem Pharmacol. 1998;56(1):121-9.

13. Ness GC, Lopez D. Transcriptional regulation of rat hepatic lowdensity lipoprotein receptor and cholesterol 7 alpha hydroxylase by thyroid hormone. Arch Biochem Biophys. 1995;323(2):404-8.

14. Harper ME, Seifert EL. Thyroid Hormone effects on mitochondrial energicts. Thyroid. 2008;18(2):145-56.

15. Targher G, Montagnana M, Salvagno G, Moghetti P, Zoppini G, Muggeo M, et al. Association between serum TSH, free T4 and serum liver enzyme activities in a large cohort of unselected outpatients. Clin Endocrinol (Oxf). 2008;68(3):481-4.

16. Chung $Y H$, Jung $S A$, Song $B C$, Chang WY, Kim JA, Song $I H$, et al. Plasma isocitrate dehydrogenase as a marker of centrilobular hepatic necrosis in patients with hyperthyroidism. J Clin Gastroenterol. 2001;33(2):118-22.

17. Diagnosis and classification of diabetes mellitus. Diabetes Care. 2014;37 Suppl 1:S81-90.

18. National High Blood Pressure Education P. In:The Seventh Report of the Joint National Committee on Prevention, Detection, Evaluation, andTreatment of High Blood Pressure. Bethesda (MD): National Heart, Lung, and Blood Institute (US); 2004.

19. Third Report of the National Cholesterol Education Program (NCEP) Expert Panel on Detection, Evaluation, and Treatment of High Blood Cholesterol in Adults (Adult Treatment Panel III) final report. Circulation. 2002;106:3143-421.

20. Boelaert K, Newby PR, Simmonds MJ, Holder RL, Carr-Smith JD, Heward JM, et al. Prevalence and relative risk of other autoimmune diseases in subjects with autoimmune thyroid disease. Am J Med. 2010;123(2):183.e1-9.

21. Kaliaperumal R, William E, Selvam T, Krishnan SM. Relationship between Lipoprotein(a) and thyroid hormones in hypothyroid patients. J Clin Diagn Res. 2014;8(2):37-9. 
22. Muratli S, Uzunlulu M, Gonenli G, Oguz A, Isbilen B. Fetuin A as a new marker of inflammation in Hashimoto thyroiditis. Minerva Endocrinol. 2015;40(1):9-14.

23. Brenta G, Vaisman M, Sgarbi JA, Bergoglio LM, de Andrada NC, Bravo PP, et al. Clinical practice guidelines for the management of hypothyroidism. Arq Bras Endocrinol Metab. 2013;57(4):265-291.

24. Delemer B, Aubert JP, Nys P, Landron F, Bouée S. An observational study of the initial management of hypothyroidism in France: the ORCHIDÉE study. Eur J Endocrinol. 2012;167(6):817-23.

25. Parle JV, Franklyn JA, Cross KW, Jones SC, Sheppard MC. Prevalence and follow-up of abnormal thyrotrophin (TSH) concentrations in the elderly in the United Kingdom. Clin Endocrinol (Oxf). 1991;34(1):77-83.

26. Dufour DR, Lott JA, Nolte FS, Gretch DR, Koff RS, Seeff LB. Diagnosis and monitoring of hepatic injury. I. Performance characteristics of laboratory tests. Clin Chem. 2000;46(12):2027-49.

27. Oh RC, Hustead TR. Causes and evaluation of mildly elevated liver transaminase levels. Am Fam Physician. 2011;84:1003-8.

28. Maximos M, Bril F, Portillo Sanchez P, Lomonaco R, Orsak B, Biernacki $D$, et al. The role of liver fat and insulin resistance as determinants of plasma aminotransferase elevation in nonalcoholic fatty liver disease. Hepatology. 2015;61(1):153-60.

29. Prati D, Taioli E, Zanella A, DellaTorre E, Butelli S, Del Vecchio E, et al. Updated definitions of healthy ranges for serum alanine aminotransferase levels. Ann Intern Med. 2002;137(1):1-10.
30. Hanley AJ, Williams K, Festa A, Wagenknecht LE, D'Agostino RB $\mathrm{Jr}$, Haffner SM. Liver markers and development of the metabolic syndrome: the insulin resistance atherosclerosis study. Diabetes. 2005;54(11):3140-7.

31. Hong Z, Yanfang J, Shumei H, Jie S, Qing G, Xiangwei F, et al. Relationship between serum aminotransferase levels and metabolic disorders in northern China. Turk J Gastroenterol. 2012;23(6):699707.

32. Saito I, Ito K, Saruta T. Hypothyroidism as a cause of hypertension. Hypertension. 1983;5(1):112-5.

33. Saito I, Saruta T. Hypertension in thyroid disorders. Endocrinol Metab Clin North Am. 1994;23(2):379-86.

34. Kubota S, Amino N, Matsumoto Y, Ikeda N, Morita S, KudoT, et al. Serial changes in liver function tests in patients with thyrotoxicosis induced by Graves' disease and painless thyroiditis. Thyroid. 2008;18(3):283-7.

35. Shantha GP, Kumar AA, Jeyachandran V, Rajamanickam D, Rajkumar K, Salim S, et al. Association between primary hypothyroidism and metabolic syndrome and the role of $C$ reactive protein: a cross-sectional study from South India. Thyroid Res. 2009;2(1):2.

36. Zhang WJ, Chen LL, Zheng J, Lin L, Zhang JY, Hu X. Association of adult weight gain and nonalcoholic fatty liver in a cross-sectional study in Wan Song Community, China. Braz J Med Biol Res. 2014;47(2):151-6. 